ARTICLE

\title{
Self-assembly and photoinduced fabrication of conductive nanographene wires on boron nitride
}

\author{
Xiaoxi Zhang (10 1,2, Fabian Gärisch (10 3 , Zongping Chen ${ }^{4,5}$, Yunbin Hu ${ }^{4,6}$, Zishu Wang ${ }^{1,2}$, Yan Wang ${ }^{1,7}$, \\ Liming Xie (1) ${ }^{8}$, Jianing Chen ${ }^{1,2}$, Juan $\mathrm{Li}^{9}$, Johannes V. Barth ${ }^{10}$, Akimitsu Narita ${ }^{4}$, Emil List-Kratochvil (1) ${ }^{3,11}$, \\ Klaus Müllen (i] ${ }^{4} \&$ Carlos-Andres Palma (i]) ${ }^{1,12 \times}$
}

\begin{abstract}
Manufacturing molecule-based functional elements directly at device interfaces is a frontier in bottom-up materials engineering. A longstanding challenge in the field is the covalent stabilization of pre-assembled molecular architectures to afford nanodevice components. Here, we employ the controlled supramolecular self-assembly of anthracene derivatives on a hexagonal boron nitride sheet, to generate nanographene wires through photo-crosslinking and thermal annealing. Specifically, we demonstrate $\mu \mathrm{m}$-long nanowires with an average width of $200 \mathrm{~nm}$, electrical conductivities of $10^{6} \mathrm{~S} \mathrm{~m}^{-1}$ and breakdown current densities of $10^{11} \mathrm{~A} \mathrm{~m}^{-2}$. Joint experiments and simulations reveal that hierarchical self-assembly promotes their formation and functional properties. Our approach demonstrates the feasibility of combined bottom-up supramolecular templating and top-down manufacturing protocols for graphene nanomaterials and interconnects, towards integrated carbon nanodevices.
\end{abstract}

\footnotetext{
${ }^{1}$ Beijing National Laboratory for Condensed Matter Physics, Institute of Physics, Chinese Academy of Sciences, Beijing 100190, PR China. ${ }^{2}$ School of Physical Sciences, University of Chinese Academy of Sciences, Beijing 100049, PR China. ${ }^{3}$ Department of Physics, Department of Chemistry \& IRIS Adlershof Humboldt-Universität zu Berlin, 12489 Berlin, Germany. ${ }^{4}$ Max Planck Institute for Polymer Research, Ackermannweg 10, 55128 Mainz, Germany. ${ }^{5}$ State Key Laboratory of Silicon Materials, Zhejiang University, Hangzhou 310027, PR China. ${ }^{6}$ College of Chemistry and Chemical Engineering, Central South University, Changsha 410083, PR China. ${ }^{7}$ School of Physics, Beijing Institute of Technology, Beijing 100081, PR China. ${ }^{8}$ National Center for Nanoscience and Technology, Beijing 100190, PR China. ${ }^{9}$ Advanced Research Institute for Multidisciplinary Science, Beijing Institute of Technology, Beijing 100081, PR China. 10 Physik-Department E20, Technische Universität München, D-85748 Garching, Germany. ${ }^{11}$ Helmholtz-Zentrum Berlin für Materialien und Energie GmbH, 14109 Berlin, Germany. ${ }^{12}$ Department of Physics \& IRIS Adlershof - Humboldt-Universität zu Berlin, 12489 Berlin, Germany. ${ }^{凶}$ email: palma@iphy.ac.cn
} 
C omplex material manufacturing through self-assembly is currently approaching atomic precision ${ }^{1,2}$, which is instrumental for the advancement of modern carbon nanodevices. In parallel to bulk preparation, surface-templated fabrication offers unique advantages for interfacial molecular architecture self-assembly and synthesis, such as rational atomistic design aided by joint computational modeling and scanning probe microscopy investigations ${ }^{3-9}$. Of fundamental interest is to establish a link between on-surface self-assembly and synthesis, and the fabrication of bottom-up structures with robust performance in the nm-to- $\mu \mathrm{m}$ scale usable for integrated carbon nanodevices. In principle, interfacial molecular architectures can be leveraged to obtain robust, functional nanoscale components via photolithography or similar top-down patterning strategies ${ }^{10}$. Towards the fabrication of precision carbon interconnects, resistors, diodes, supercapacitors, and other electronic components $^{11}$, it is favorable to crosslink supramolecular architectures into nanosheets or nanowires 12,13 , and control their processing by, e.g., ultra-fast Joule heating ${ }^{14}$ or thermal conversion ${ }^{15,16}$. However, atomic precision and functionality in the context of nanodevices remain largely unexplored in interfacial molecular architectures. Regarding atomic precision, a major challenge is to control the organization of the architecture to induce advantageous covalent reaction pathways ${ }^{17-23}$ using for example a variety of homolytic cleaving groups to induce crosslinking10,20,21,24,25. Concerning functionality, two major obstacles regarding applications are moderate device performances and processing conditions incompatible with nanofabrication. For modern nanomanufacturing, it is desirable to template supramolecular architectures in device configurations of choice, and react them in situ to reach the target technology, such as broadband and conductive (e.g., $\left.10^{7} \mathrm{~S} \mathrm{~m}^{-1}\right)^{26}$ nanoscale interconnects together with state-of-the-art logic elements (e.g., transistor or quantum circuits). Overall, an important frontier of atomically-precise bottom-up science is to attempt to engineer nanodevices, by stimuli-induced crosslinking or thermal annealing of increasingly regular supramolecular architectures $18,25,27,28$ at device interfaces.

Herein we adopt a surface-templated approach to fabricate and monitor supramolecular nanoarchitectures on hexagonal boron nitride (BN), and their conversion into highly conducting nanowires $\left(10^{6} \mathrm{~S} \mathrm{~m}^{-1}\right.$, Fig. 1) between gold electrodes in situ. In an ultra-high vacuum (UHV) environment, we thermally sublimated precursor molecules, brominated anthracene carboxylic acid derivative $\mathbf{1}$ on $\mathrm{BN}$, to guide hydrogen-bonding and hierarchical assembly of the molecules ${ }^{29,30}$. Joint molecular dynamics (MD), scanning tunneling microscopy (STM) and atomic force microscopy (AFM) studies reveal that the molecular units form a periodic pattern according to the expected $\pi$-stacking motif with $d=3.5 \pm 0.2 \AA$ at the $\mathrm{BN} / \mathrm{Cu}(111)$ interface. Supramolecular architectures grow upward in the direction perpendicular to the surface of the $\mathrm{BN}$ substrate, and supramolecular nanowires evolve with a micrometer length, as characterized by scanning electron microscopy (SEM). After their formation, ultraviolet (UV) light is employed to induce debromination of supramolecular structures of $\mathbf{1}$ to form species $\mathbf{2}$, monitored by matrix-assisted laser desorption ionization (MALDI) mass spectrometry (MS) ${ }^{24}$. Species 2 engages subsequently in dehydrogenative coupling, affording nanographene wires, whereby Raman spectroscopy indicates further dehydrogenative chemical conversions following $1273 \mathrm{~K}$ annealing, providing highly regular nanowires. Mean conductivities of $(1.6 \pm 2.0) \times 10^{6} \mathrm{~S} \mathrm{~m}^{-1}(19.7 \pm 7.3 \Omega$ per $\mu \mathrm{m}$ of a $200 \mathrm{~nm}$ diameter nanowire) and breakdown current densities of $(1.6 \pm 1.9) \times 10^{11} \mathrm{~A} \mathrm{~m}^{-2}$ further substantiate successful nanowire synthesis on insulators.

\section{Results \& discussion}

Simulations and STM of interfacial self-assembly. Brominated anthracene was successfully employed for on-surface synthesis of polyanthrenes and ultra-narrow graphene nanoribbons ${ }^{31}$. Nanographene formation involves three steps: debromination, free radical-radical coupling, and thermal dehydrogenation ${ }^{32,33}$. On metal surfaces, the success of the reaction relies on efficient diffusion and low steric hindrance between the radical intermediates $^{32,33}$. On insulators, the debromination can be assisted by light, but to date yielded amorphous materials ${ }^{24}$. Notwithstanding this, ordered assemblies with closely packed bromines can promote radical-radical coupling ${ }^{24,25}$. Precursor 1, 9,10-dibromo-anthracene-2-carboxylic acid, favors expression of supramolecular dimers via carboxylic acid H-bonds, $(\mathbf{1})_{2}$, providing the constituents for robust $\pi$-stacking complexes (Fig. 2a). Following this strategy, adjacent $\mathrm{Br}$ atoms can be abstracted by the stimulation with UV light, potentially promoting directional radical-radical coupling 24,25 . To understand the propensity of the precursors towards hierarchical assembly via $\mathrm{H}$-bonding and subsequent $\pi$-stacking, several CHARMM force field ${ }^{34}$ molecular dynamic (MD) simulations were performed for ten randomly distributed molecules in vacuo. After 1-ns simulations at temperatures between $370 \mathrm{~K}$ and $400 \mathrm{~K}$, an average number of six $\pi$-stacks indicates the formation of a $4 \bullet(1)_{2}$ supramolecular stacks (Fig. 2b). One representative $4 \bullet(1)_{2}$ stack extracted from the MD simulations and optimized by self-consistent charge, dispersion corrected, third-order density functional tight binding (SCC DDFTB3) employing the DFTB + package ${ }^{35}$ is depicted in Fig. 2c. The cluster consists of two stacks of cis- $(\mathbf{1})_{2}$ and two of trans- $(\mathbf{1})_{2}$. The $\pi$-stack distances amount to $d=3.4 \AA$ for the Grimme implementation of dispersion correction ${ }^{36}$ (D3) and $d=3.6 \AA$ for a Lennard Jones (LJ) implementation. These two methods usually correspond to lower and upper estimates, respectively, of common stacking distances 37,38 .

To study self-assembly and crosslinking under equivalent conditions, a monolayer of 1 (cf. Supplementary Synthesis, and Supplementary Figs. 1 and 2) was sublimated (30 min, $433 \mathrm{~K}$ ) under UHV $\left(10^{-9}\right.$ mbar) on top of a model substrate for inert self-assembly ${ }^{39}$ : An hexagonal $\mathrm{BN}$ monolayer on $\mathrm{Cu}(111)^{40}$. The sublimation temperatures employed are much below the decomposition temperature of $\mathbf{1}$ as measured by thermal gravimetric analysis $(>677 \mathrm{~K})$. Cryogenic STM data (Fig. 2d) reveals the supramolecular structure is characterized by rows with an interrow periodicity a and intrarow periodicity $\mathbf{c}=(3.5 \pm 0.2)$ $\AA$. The interrow distance is closer than previously reported anthracene carboxylic acid structures $(\sim 3.9 \AA)^{41}$. For straight patterns, a amounts to $6.4 \AA$. To elucidate probable molecular arrangements, departing from a supramolecular dimer $(\mathbf{1})_{2}$, we built a disordered cluster featuring 45 , upstanding, trans- $(\mathbf{1})_{2}$ dimers on BN (Fig. 2e). After a few ns of MD simulations at $295 \mathrm{~K}$ (Fig. 2f), the cluster self-assembled into a brick structure ${ }^{42}$ with $\mathbf{c}=3.7 \AA$ and interrow distances of $\mathbf{a}=7.5 \AA$, in close agreement with experimental data. We assign the $\sim 1 \AA$ interrow discrepancy with experiment to the finite size of our cluster, since larger clusters or structures induce denser configurations. It is worth noting that lower deposition times result in flat-on molecular arrangements on the $\mathrm{BN} / \mathrm{Cu}(111)$ substrate, as shown in the STM data inset in Fig. 1 and Supplementary Fig. 3. Polymorphic domains in large area STM surveys (Supplementary Fig. 4) reveal isolated rows, without neighbors or with odd-number of neighboring rows, which cannot account for in-plane $\mathrm{H}$-bond formation (cf. Supplementary Fig. 3). Hence, the upstanding $\pi$ stacked $(\mathbf{1})_{2}$ rows are likely held together by interrow $v d W$ interactions only. The absence of interrow distances comparable to the H-bonded cis or trans interdimer distance of $\sim 10-14 \AA$ additionally substantiates this conclusion (cf. dimer length in 


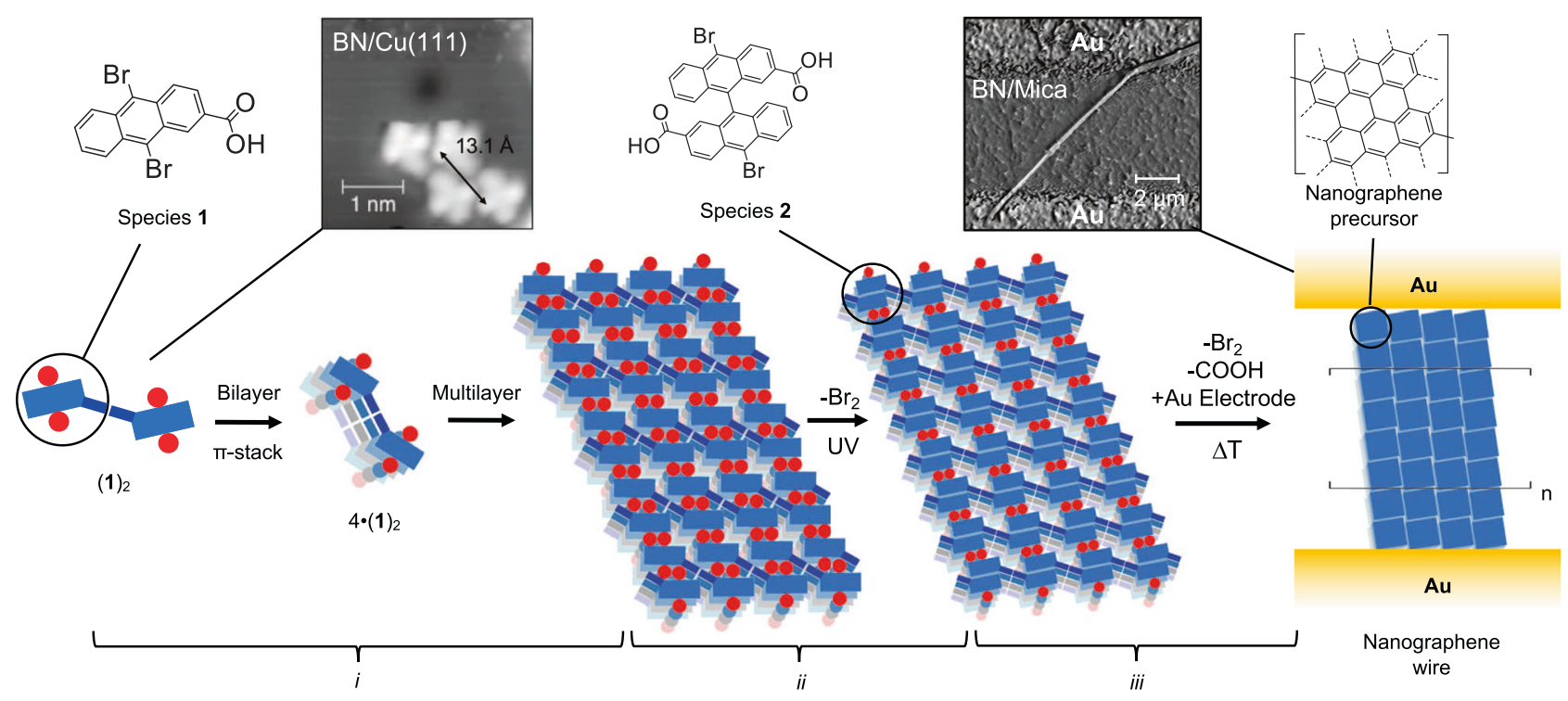

Fig. 1 Hierarchical self-assembly, crosslinking and thermal conversion of supramolecular structures. Species 1 undergoes supramolecular H-bond dimerization, $\pi$-stacking and $v d W$ layered growth into supramolecular nanowires (i). Species $\mathbf{2}$ can be formed by ultraviolet (UV) photo-crosslinking within the supramolecular architectures formed by species $\mathbf{1}$ (ii), and subsequently undergoes thermal dehydrogenation to nanographene precursors and thermal crosslinking into nanographene networks (iii). The so-formed nanographene wires are defined by the nanographene material retaining their supramolecular architecture wire-like scaffold. The inset in (i) shows the scanning tunneling microscopy (STM) data of four flat-on molecules on the BN/Cu(111) surface, forming two (1) $)_{2}$ supramolecular dimers, held together by an interrow hydrogen bond. The inset in (iii) shows the scanning electron microscopy (SEM) of the final nanographene wire between electrodes. STM parameters: $I_{t}=80 \mathrm{pA}, V_{s}=1.0 \mathrm{~V}$. SEM parameters $2 \mathrm{kV}, I=10 \mu \mathrm{A}$.

Fig. 1 inset with Fig. 2c and Supplementary Figs. 3 and 4). This type of upstanding molecular orientation is possible for multilayers of acenes ${ }^{43-45}$ and benzothiophenes ${ }^{46}$ on graphene and $\mathrm{BN}$, where interrow distances are close to $6 \AA$. These exhaustive STM and MD simulations identify three hierarchical levels of assembly: carboxylic acid dimer formation, intrarow $\pi$-stacking, and interrow $v d W$ interactions.

Simulations and characterization of supramolecular nanowires. Microsecond-long simulations of 576 molecules at $350 \mathrm{~K}$ were employed to complementarily study $(\mathbf{1})_{2}$ multilayered stacking perpendicular to the substrate surface, that is, in three dimensions (3D). The simulations reveal that brick-packed layers can grow in a layer-by-layer manner, stacking in $3 \mathrm{D}$, with a triclinic unit cell extracted from the steepest descent optimization of the $(\mathbf{1})_{2}-(\mathbf{1})_{2}$ interlayer motif amounting to $\mathbf{a}=9.0 \AA, \mathbf{b}=21.3 \AA$, $\mathrm{c}=4.4 \AA$ with $\alpha, \beta, \gamma=63^{\circ}, 68^{\circ}, 80^{\circ}$, respectively (Fig. $3 \mathrm{a}, \mathrm{b}$ ). Preparations employing higher deposition fluxes $(30 \mathrm{~min}$ at $\left.453 \mathrm{~K}, 10^{-9} \mathrm{mbar}\right)$ at a $\mathrm{BN} / \mathrm{Cu}(111)$ substrate temperature of 353-373 K evidence layered growth (Fig. 3c), whereby the STM data in Fig. $3 c$ depicts molecular islands associated with stacked layers on top of each other. The line profile in Fig. 3d shows an apparent height of the first step of $21 \AA$. This height is close to the $\mathrm{Br}$-substrate distance of the first layer, amounting to $\mathbf{h}=19.5 \AA$ (triangle, Fig. 3b) and is similar to the projection of the length of $(\mathbf{1})_{2}$ in Fig. $3 a$ to the substrate $(15.1 \AA)$, plus the adsorption height. Considering an adsorption height of $3.4 \AA$, the discrepancy $(\Delta h=19.5 \AA-18.5 \AA)$ is assigned to a steeper first layer on the surface as compared to the unit cell. The interrow distances (blue arrows, Fig. 3b) and height of the first step are too far apart and high, for anything but two upstanding $(\mathbf{1})_{2}$, in accordance with Fig. 2 data. However, the STM apparent height difference between the first and second layer ( $8 \AA$ Fig. $3 \mathrm{~d}$, cf. Supplementary Fig. 5) is lower than the simulated bilayer height in Fig. $3 b(15.1 \AA)$. Because the rows of the second layer are very similar to those of the first layer, we assign the height discrepancy to electronic or vibronic tunneling effects. Note how the rows are oriented perpendicular to the substrate's high symmetry directions, an effect which is reproduced in the simulations due to bromine and hydrogen preferring the hollow sites of the honeycomb lattice in molecular mechanics.

In further studies below, we employed longer deposition times aiming at slow, multilayered growth of supramolecular architectures. For these studies the substrate $\mathrm{BN}$ was transferred from copper to mica sheet. After $60 \mathrm{~min}$ deposition, supramolecular nanowires of $\mathbf{1}$ are further evidenced in AFM data (Fig. 3e-h). Height profiles are shown along the tentative growth directions, where step heights and lengths of $\sim 3 \mathrm{~nm}$ can be resolved (Fig. 3e, f). Photoluminescence (PL) microscopy reveals that the supramolecular structures form $\sim 20$ - $\mu \mathrm{m}$-long nanowires on $\mathrm{BN}$ surface upon $60 \mathrm{~min}$ deposition, featuring apparent, diffractionlimited widths of $\sim 1 \mu \mathrm{m}$ (Fig. $3 \mathrm{~g}$, i). The PL identifies the supramolecular peak emission at $480 \mathrm{~nm}$ upon $350 \mathrm{~nm}$ excitation. SEM shows that the typical nanowire width amounts to 80-400 nm for $>5 \mu \mathrm{m}$-long nanowires before (Supplementary Fig. 7a, c) and after (Fig. 3j, Supplementary Fig. 7b, d) UV induced crosslinking, by means of an OSRAM UV-Vis lamp $\left(\lambda_{\max } 366 \mathrm{~nm}, 250 \mathrm{~W}\right)$ for $12 \mathrm{~h}$ under UHV. Inspection of SEM data sets reveal that nanowires are needle-like, become thinner at the tip, and hang away from the BN substrate. These observations evidence surface-templated nanowires, tentatively promoted by island nucleation in Fig. $3 c^{47}$.

Photo-crosslinking and thermal conversion into nanographene wires. Further observations were conducted to address the supramolecular nanowire photo-crosslinking, with key results depicted in Fig. 4. Ex-situ MALDI-MS measurements provide evidence for dimerization of $\mathbf{1}$ into species $\mathbf{2}$ after UV irradiation whereby the MS main isotopic distribution is identified as crosslinked species 2, $\mathrm{C}_{30} \mathrm{H}_{16} \mathrm{Br}_{2} \mathrm{O}_{4}$ (calc. $m / z=597.94$, exp. $m / z=597.96$, Fig. 4b). Simultaneously, MS signals of the tetrabrominated species $\mathrm{C}_{30} \mathrm{H}_{16} \mathrm{Br}_{4} \mathrm{O}_{4}$ (calc. $m / z=755.78$ ) are absent, which exclude supramolecular complexes or cycloaddition between the anthracene cores prior to debromination. 

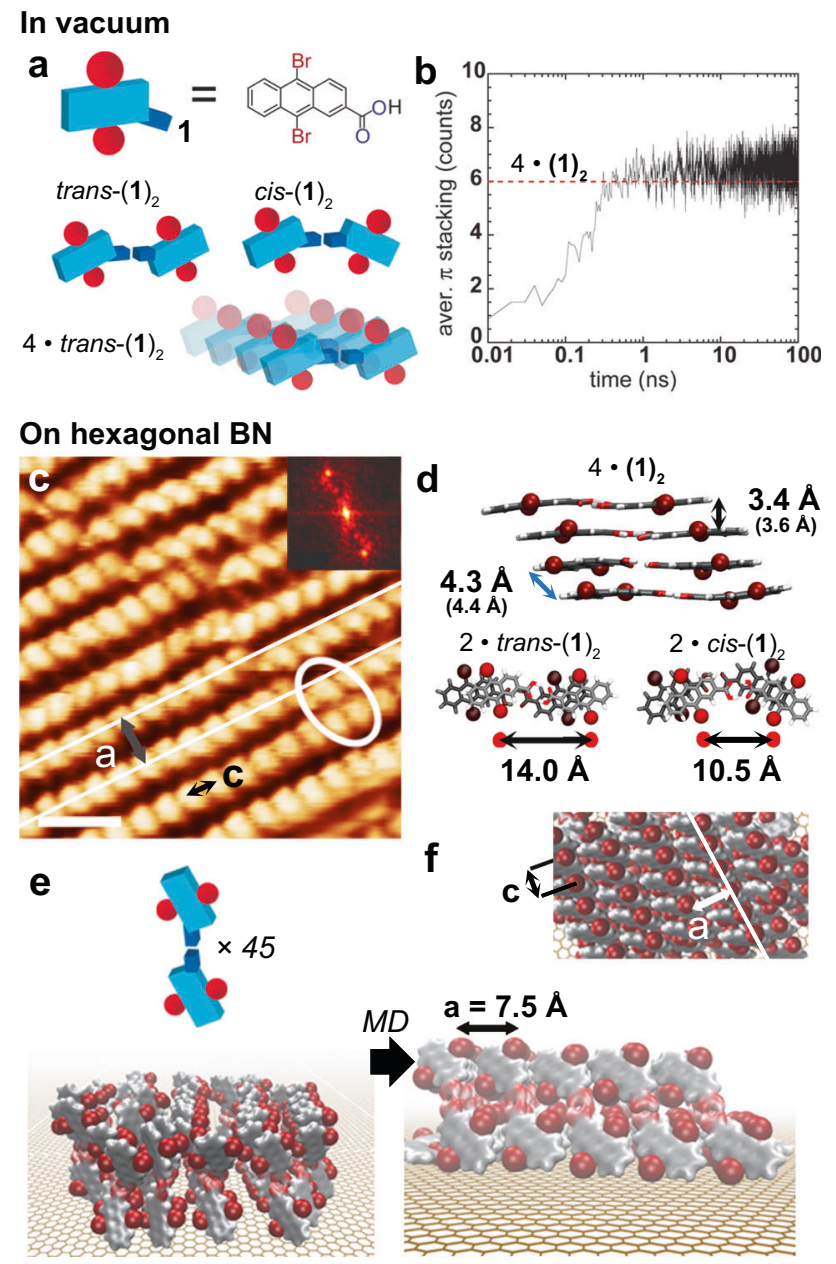

Fig. 2 Simulations and scanning tunneling microscopy detail of the firstlayer self-assembled stacks of $\left(\mathbf{1}_{\mathbf{2}}\right.$ on boron nitride. a $\mathrm{H}$-bond and $\pi$-stacking of $\mathbf{1}$ may promote densely packed rows. $\mathbf{b}$ In vacuo molecular dynamic (MD) simulations of ten molecules of $\mathbf{1}$ in a $125 \mathrm{~nm}^{3}$ box show ultrafast propensity towards hydrogen bond dimerization into $(\mathbf{1})_{2}$ and $\pi$-stacking: After $1 \mathrm{~ns}$ the number of $\pi$-stacks (6-8) indicate the formation of $4 \bullet(\mathbf{1})_{2}$ stacks. c Ultra-high vacuum scanning tunneling microscopy (UHV STM) data at $20 \mathrm{~K}$ reveals supramolecular structures with periodicity $\mathrm{c}=(3.5 \pm 0.2) \AA$ on $\mathrm{BN} / \mathrm{Cu}(111)$. Scale bar $1 \mathrm{~nm}$. STM parameters $I_{t}=80$ $\mathrm{pA}, V_{s}=1.0 \mathrm{~V}$. In the straight pattern the interrow distance is $\mathrm{a}=6.4 \AA$. d Dispersion-corrected density functional tight binding (DFTB) optimization of a $4 \bullet(\mathbf{1})_{2}$ stack extracted from the MD simulations, containing two stacks of trans- $(\mathbf{1})_{2}$ and two of cis- $(\mathbf{1})_{2}$ with $d=3.4 \AA$. The numbers in parenthesis refer to calculations employing a Lennard-Jones potential. e, $\mathbf{f}$ Molecular dynamics of 45 upstanding edge-on species of $(\mathbf{1})_{2}$ on $B N$ reveals selfassembly in regular patterns with an interrow distance of $a=7.5 \AA$, $c=3.7 \AA$. Inset: Top-view of the assembly after $10 \mathrm{~ns}$.

To rationalize the debromination and dimerization coupling process into species 2, we perform DFTB-MD simulations at $298 \mathrm{~K}$ by artificially removing $\mathrm{Br}$ atoms between $(\mathbf{1})_{2}-(\mathbf{1})_{2}$ interlayers. This approximation considers that $\mathrm{C}-\mathrm{Br}$ photodissociation begins to occur at $\sim 0.1 \AA$ from the equilibrium distance ${ }^{24}$, so that the crystalline structure is not significantly altered when $\mathrm{Br}$ (or e.g., $\mathrm{Br}_{2}$ or $\mathrm{HBr}$ ) dissociates and desorbs through vacancies in the molecular architecture during the $12 \mathrm{~h}$ of UV treatment under $\mathrm{UHV}$ conditions. In the simulations, $\mathrm{C}-\mathrm{C}$ coupling is observed for interlayers with a twist angle of $90^{\circ}$ (Fig. 4a). We note that $\mathrm{C}-\mathrm{C}$ coupling for different twist angles is possible, but was not investigated in detail due to the related increase of the simulation supercell size. Importantly, polarized Raman (for $\lambda_{0}=532 \mathrm{~nm}$ ) of UV irradiated structures shows a high degree of anisotropy, pointing towards a topochemical effect of UV irradiation (Fig. 4d, Supplementary Fig. 9a, b, black line). The anthracene peak near $1400 \mathrm{~cm}^{-1}$ sharpens after irradiation, contrary to its absence in anthracene photocycloaddition experiments ${ }^{48}$.

DFTB-MD depicts a tentative mechanism whereby heating of the unit cell in Fig. 4a to $1273 \mathrm{~K}$ affords $\mathrm{C}-\mathrm{H}$ bond breaking (Fig. 4c), and nanographene formation (Supplementary Fig. 6). Interestingly, we find that annealing supramolecular nanowires to $1273 \mathrm{~K}$ for $30 \mathrm{~min}$ is possible by fixing the nanowires between gold electrodes (Fig. 4e-g, Supplementary Figs. 12 and 13). It is worth mentioning that the nanowire will evaporate if annealed without UV treatment (Supplementary Fig. 14). Raman spectroscopy of the UV irradiated 1 on $\mathrm{BN} / \mathrm{mica}$ annealed $30 \mathrm{~min}$ to $1273 \mathrm{~K}$ reveals a $70 \mathrm{~cm}^{-1} \mathrm{FWHM}$ signal centered at $1602 \mathrm{~cm}^{-1}$ for $\lambda_{0}=532 \mathrm{~nm}$ excitation (Fig. $4 \mathrm{~d}$ magenta) with a side peak at $\sim 1589 \mathrm{~cm}^{-1}$ close to the graphene $\mathrm{G}$ peak $\mathrm{E}_{2 \mathrm{~g}}$ mode ${ }^{49}$ at $\lambda_{0}=532 \mathrm{~nm} \sim 1580 \mathrm{~cm}^{-1}$. A G peak FWHM $<100 \mathrm{~cm}^{-1}$ together with a notably weak signal near the $D$ peak $^{50}\left(\sim 1410 \mathrm{~cm}^{-1}\right.$, $\mathrm{FWHM}=60 \mathrm{~cm}^{-1}, \mathrm{D} / \mathrm{G}$ intensity ratio $\left.<0.3\right)$ is contraindicative of amorphous carbon or high $\mathrm{sp}^{3}$ carbon content ${ }^{51}$ at $\lambda_{0}=532 \mathrm{~nm}$. Instead, the weak $\mathrm{G}$ peak dispersion $\left(1610 \mathrm{~cm}^{-1}\right.$ at $\lambda_{0}=325 \mathrm{~nm}$, Supplementary Fig. 11c) and background shape around the $2 \mathrm{D}$ and $2 \mathrm{G}$ peaks, supports strained, randomly stacked, or n-doped graphene possibly connected or intercalated by oxygen or bromine atoms $\mathrm{s}^{51,52}$. For example, absence of $2 \mathrm{D}$ peaks and a signal at $1586 \mathrm{~cm}^{-1}$ accompanied by a broad $1500 \mathrm{~cm}^{-1}$ background has been reported ${ }^{53}$ for a carboxylated nanographene $\mathrm{e}^{54}$ with $60 \mathrm{sp}^{2}$ carbons. We conclude that the nanowires consist of crosslinked, distinctly-shaped pieces of nanographenes, departing from dehydrogenated species $\mathbf{2}$ observed in simulations (Fig. 4c). To illustrate this, we summarize in Supplementary Fig. 10 the evolution of the broad $1620 \mathrm{~cm}^{-1}$ signal in the powder of $\mathbf{1}$ and upon self-assembly and UV treatment, from a sharp carbon $\mathrm{sp}^{2}$ signal at $1610 \mathrm{~cm}^{-1}$ indicative of a highly regular crosslinked material, to a broad signal below $1600 \mathrm{~cm}^{-1}$ after annealing.

After $1273 \mathrm{~K}$ annealing, PL microscopy reveals PL intensity quenching of the nanowire (close to $480 \mathrm{~nm}$ at $350 \mathrm{~nm}$ excitation Fig. 4e, f), in agreement with the PL spectroscopy (Supplementary Fig. 15) and a controlled dehydrogenation ${ }^{33}$. Electrical characterization of nanowires at room temperature and under argon flow reveal resistances of $(118 \pm 44) \Omega$ for electrode-electrode distances of ca. $6 \mu \mathrm{m}$, which correspond to a conductivity of $(1.6 \pm 2.0) \times 10^{6} \mathrm{~S} \mathrm{~m}^{-1}$ $(19.7 \pm 7.3 \Omega$ per $\mu \mathrm{m}$ for a $200 \mathrm{~nm}$ diameter nanowire, Fig. $4 \mathrm{~h})$, given an approximated cylindrical nanowire diameter of $(200 \pm 120)$ nm. We note that slight deviations from a linear ohmic behavior have been observed at very low $(\sim \mathrm{nA})$ and high currents $(\sim \mathrm{mA})$, which is attributed to initial Joule heating effects and fatigue at high current densities, respectively. The attained conductivity value of our nanowires is found to be at the order of magnitude of some metal alloys (Galinstan, Constantan) and it is therefore higher than that of CVD or electro-spun carbon nanofibers $\left(10 \mathrm{~S} \mathrm{~m}^{-1}\right.$ to $3 \times 10^{2} \mathrm{~S} \mathrm{~m}^{-1}$, or equivalently $1.1 \times 10^{5} \Omega$ to $2.6 \times 10^{6} \Omega$ per $\mu \mathrm{m}$ for $200 \mathrm{~nm}$ diameter carbon nanofibers $)^{55,56}$ and highly ordered graphene fibers or nanotube bundles $\left(\sim 10^{5} \mathrm{~S} \mathrm{~m}^{-1}, 3.1 \times 10^{-2} \Omega\right.$ per $\mu \mathrm{m}$ length, for $20 \mu \mathrm{m}$ diameter graphene fibers $)^{16,57}$. The conductivity of the reported nanographene wires is close to that of graphene formed on the etched sidewalls of silicon carbide, which can achieve ballistic transport up to 10 microns length (i.e., $300 \mathrm{~nm}$ width ballistic transport graphene ribbons have a conductivity of $\sim 2 \times 10^{6} \mathrm{~S} \mathrm{~m}^{-1}$, $\sim 10^{2} \Omega$ per $\mu \mathrm{m}$ length of ribbon $)^{58,59}$ and approaches the conductivity of Ag nanowires $\left(\sim 4 \times 10^{7} \mathrm{~S} \mathrm{~m}^{-1}, 0.2 \Omega\right.$ per $\mu \mathrm{m}$ for a $200 \mathrm{~nm}$ diameter nanowire $)^{60}$ and nanotube- $\mathrm{Cu}$ nanowires ${ }^{26}$. 


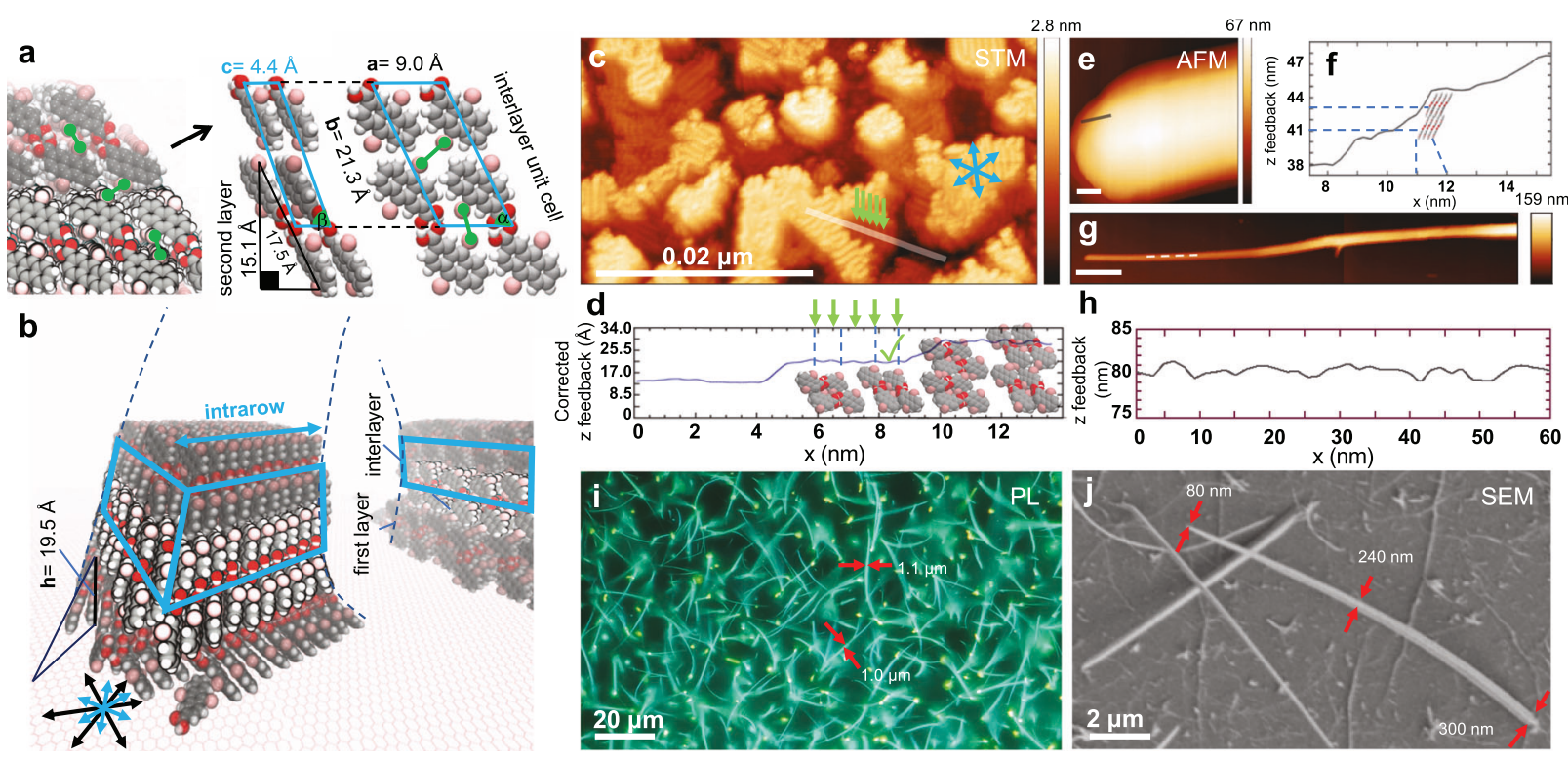

Fig. 3 Simulations, analysis and ultraviolet irradiation of supramolecular nanowires. a A simulated interlayer $(\mathbf{1})_{2}-(\mathbf{1})_{2}$ unit cell on top of the first-layer and, $\mathbf{b}$ overview of ns-long molecular dynamics at $350 \mathrm{~K}$ depicting nanowire pillars on hexagon-boron nitride (BN). Molecules loosely adsorbed on the substrate have been removed for clarity. The cyan boxes depict the interlayer region supercells, and the intrarow direction (blue arrow) is shown perpendicular to the substrates' high symmetry directions (black arrows). c Ultra-high vacuum scanning tunnel microscopy (UHV STM) data at $~ 20 \mathrm{~K}$ of islands of $\mathbf{1}$ after 30 min multilayer deposition on BN/Cu(111). The blue arrows depict the row directions. $\mathbf{d}$ The apparent height profile along two strata in $\mathbf{c}$. Standing $(\mathbf{1})_{2}$ dimers on the substrate are shown to scale. The rows are independent of each other and the average interrow separation (green arrows) is $<1 \mathrm{~nm}$, similar to the interrow parameter in Fig. 2 . STM parameters $I_{t}=80 \mathrm{pA}, V_{s}=800 \mathrm{mV}$. e, $\mathbf{g}$ Atomic force microscopy (AFM) detail of tip of a nanowire after $60 \mathrm{~min}$ multilayer deposition on BN/mica. The scale bars in $\mathbf{e}$ and $\mathbf{g}$ are $7 \mathrm{~nm}$ and $50 \mathrm{~nm}$, respectively. $\mathbf{f}$ The profile of $\mathbf{e}$ depicts eight stacked $(\mathbf{1})_{2}$ shown to $\mathbf{z}$-scale and $\sim 2 \mathrm{~nm}$ height variations along the wire. $\mathbf{h}$ The profile of $\mathbf{g}$ depicts $\sim 2 \mathrm{~nm}$ height variations along the wire. i Photoluminescence $(\mathrm{PL})\left(\lambda_{\mathrm{ex}}=350 \mathrm{~nm}\right)$ microscopy showing supramolecular nanowires after 60 min multilayer deposition on BN/mica and corresponding diffraction-limited widths (red arrows). $\mathbf{j}$ Scanning electron microscopy of the structures after ultraviolet (UV) irradiation, cf. structures before irradiation Supplementary Fig. 7. SEM parameters $1 \mathrm{kV}, I=10 \mu \mathrm{A}$.
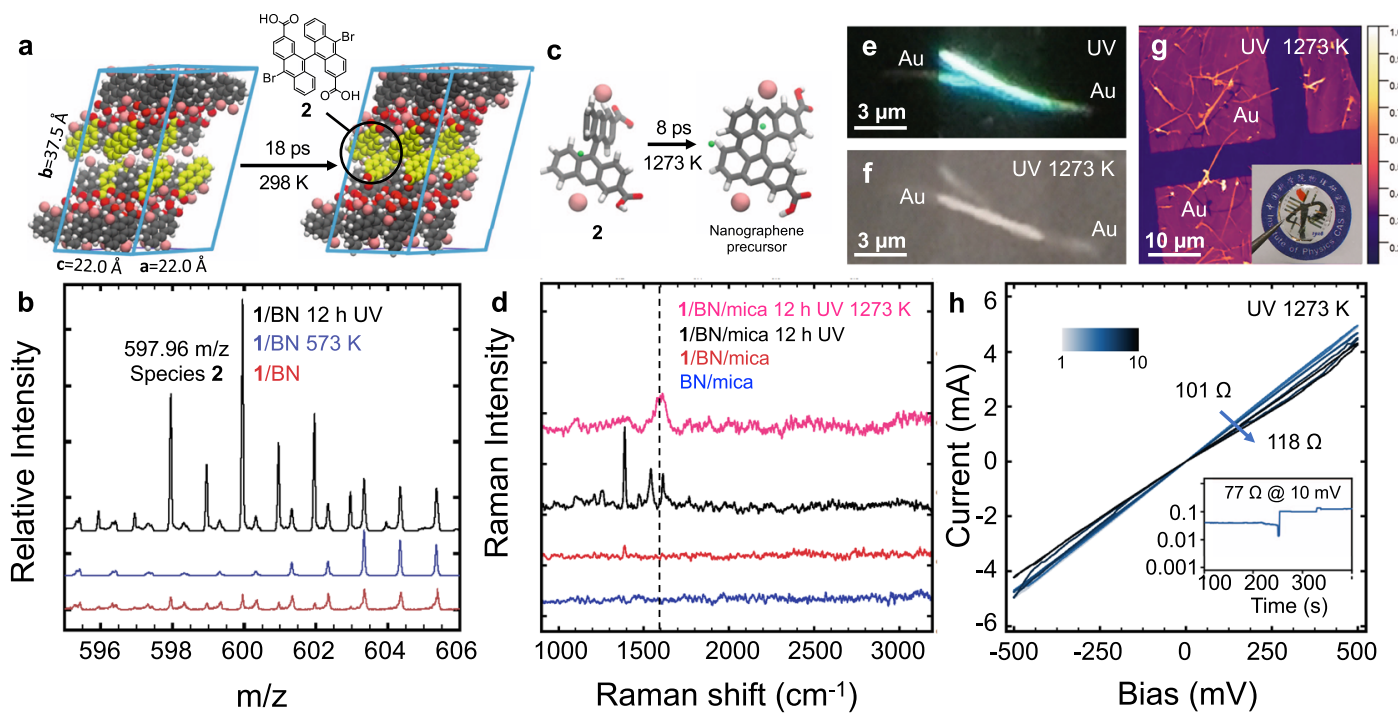

Fig. 4 Photo-crosslinking and annealing into nanographene wires. a DFTB-MD simulated C-C bond formation at 298 K after debromination. b Matrixassisted laser desorption ionized mass spectrum (MALDI-MS) of $\mathbf{1}$ after ultraviolet (UV) irradiation on boron nitride (BN) on mica shows formation of species $\mathbf{2}(\mathrm{m} / z=597.96)$. Species $\mathbf{2}$ is not detected when the sample is annealed at $573 \mathrm{~K}$ under argon without UV treatment. c The C-H bond breaking DFTB-MD simulation during the high temperature transformation of the supercell in a followed by $\mathrm{C}-\mathrm{C}$ bond formation. Dissociating $\mathrm{H}$ atoms are shown in green. d Raman spectroscopy of an individual nanowire. The $1389 \mathrm{~cm}^{-1}$ anthracene signal intensifies after UV irradiation. After $1273 \mathrm{~K} 30 \mathrm{~min}$ annealing, the $1541 \mathrm{~cm}^{-1}$ signal which appears after UV irradiation weakens, and the $1602 \mathrm{~cm}^{-1}$ peak (black dash line) broadens. $\lambda_{\mathrm{ex}}=532 \mathrm{~nm}$ and $2 \mathrm{~mW}, 60 \mathrm{~s}$ integration time. e, $\mathbf{f}$ Photoluminescence ( $\mathrm{PL}$ ) microscopy $(480 \mathrm{~nm}$ emission at $350 \mathrm{~nm}$ excitation) of the nanowire between two gold electrodes after photo-crosslinking, and after $1273 \mathrm{~K} 30 \mathrm{~min}$ annealing. $\mathbf{g}$ Laser confocal microscopy bright field image of a nanowire. The inset shows a photo of $\mathbf{1} / \mathrm{BN} / \mathrm{mica}$ sample with Au electrodes. $\mathbf{h}$ High current I-V characteristics of a single $\sim 200 \mathrm{~nm}$-wide nanowire reveals a resistance of $101 \Omega$ which increases in time. The inset shows the junction stability at $10 \mathrm{mV}$ and low currents. 
We found that operating nanowires at higher biases than $0.5 \mathrm{~V}$ can lead to enhanced thermal annealing effects further increasing the conductivity. Yet, in most cases the nanowires are damaged, with a breakdown current density of $(1.6 \pm 1.9) \times 10^{11} \mathrm{~A} \mathrm{~m}^{-2}$ (Supplementary Fig. 16). This breakdown current density is placed between carbon nanofibers $\left(\sim 10^{10} \mathrm{~A} \mathrm{~m}^{-2}\right)^{26}$ and graphene nanoribbons $\left(\sim 10^{12} \mathrm{~A} \mathrm{~m}^{-2}\right)^{61}$, in agreement with stacked nanographenes. The advantage of record conductivities and currents density measured in bottom-up nanofabricated structures is multifold: Functional properties, (current) annealing effects, transport physics, and circuitry can be tailored by means of atomic dopants in the precursors, multicomponent self-assembly and photolithography.

In summary, we have studied the self-assembly, photocrosslinking, thermal conversion and conductivity of anthracene derivatives on $\mathrm{BN}$ substrates. The supramolecular architecture precursor self-assembles with four hierarchical levels of interplay: carboxylic acid dimer formation, $\pi$-stacking, interrow and interlayer $v d W$ interactions, affording 80 to $400 \mathrm{~nm}$-wide supramolecular nanowires. Joint experiment and simulations provide evidence for the formation of nanographene upon UV irradiation and thermal conversion between gold electrodes, resulting in covalently bonded, robust nanographene wires with conductivities of $(1.6 \pm 2.0) \times 10^{6} \mathrm{~S} \mathrm{~m}^{-1}$ approaching those of noble metal nanowires. Our work helps bridge the gap between basic research in on-surface self-assembly and integrated carbon nanodevices, promoting the development of bottom-up templated molecular nanomaterials and crosslinkable organic frameworks (XOFs) with top-down atomic crosslinking precision ${ }^{62}$ directly at device interfaces.

\begin{abstract}
Methods
Substrate preparation. The BN surface was prepared either in situ by chemical vapor deposition (CVD) of borazine on $\mathrm{Cu}(111)$ under UHV following a protocol described ${ }^{40}$ for the scanning tunneling microscopy experiments, or transferred from commercial BN on $\mathrm{Cu}$ foil (Graphene Supermarket) to mica (Changchun Taiyuan Company). First, we coated polydimethylsiloxane (PDMS) on $\mathrm{BN} / \mathrm{Cu}$, followed by ammonium persulfate solution for $8 \mathrm{~h}$. After that, the copper was completely etched, and the BN on PDMS was picked up with the mica sheet. After cleaning in deionized water and drying for $1 \mathrm{~h}$, we exfoliated the PDMS very slowly. It is important to note that $\mathrm{BN}$ on mica substrates were annealed to $673 \mathrm{~K}$ for $1 \mathrm{~h}$ under air to remove PDMS residues ${ }^{63}$ until no fluorescence was detected from the substrate. Subsequently the substrates were transferred to UHV.
\end{abstract}

Sample preparation. The DBA-COOH molecule 1 was sublimated to the substrate under UHV $\left(5 \times 10^{-9} \mathrm{mbar}\right)$ between $433-453 \mathrm{~K}$ for $30 \mathrm{~min}$ to prepare a monolayer or multilayer of the molecules, and $60 \mathrm{~min}$ to prepare the nanowires. The filament of the evaporator was preheated for $15 \mathrm{~min}$. After sublimation, all samples were flash annealed at $352 \mathrm{~K}$ before characterization and irradiated by a $250 \mathrm{~W}$ OSRAM HWL lamp without its outer bulb (featuring $\lambda_{(\mathrm{UV})}=254,266,303,313$, $335,366 \mathrm{~nm})$ under UHV for $12 \mathrm{~h}\left(10^{-9} \mathrm{mbar}\right)$. For the last step, $\mathrm{Au}(111)$ electrode pads (30-200 nm thickness) were evaporated. We used a SKY Technology DZ-300 thermal evaporator to evaporate the Au electrodes. The electrode was evaporated through a shadow mask under $5 \times 10^{-7}$ mbar. The sample was then annealed to $1273 \mathrm{~K}$ via e-beam heating, employing $600 \mathrm{~V}$ sample voltage for $30 \mathrm{~min}$ maintaining UV irradiation at high vacuum $\left(10^{-8} \mathrm{mbar}\right)$.

Scanning probe microscopy. Scanning tunneling microscopy (STM) was carried out using a CreaTec STM operating between $6 \mathrm{~K}$ and $20 \mathrm{~K}$ under ultra-high vacuum conditions. Molecules were deposited using a quartz container held between $433 \mathrm{~K}$ or $453 \mathrm{~K}$ while the $\mathrm{BN} / \mathrm{Cu}(111)$ substrate ${ }^{40}$ and the sample was kept at $293 \mathrm{~K}$ followed by annealing steps described in the main text. All STM data was recorded in constant current mode. The STM images in Fig. 3 were processed using the Gwyddion software 64 , and the $\mathrm{Z}$ feedback corrected by a factor of $0.77 / 3.5$, where $0.77 \AA$ is the apparent STM height of the dimer on $\mathrm{BN} / \mathrm{Cu}(111)$ in Fig. 1 (inset in $i$ ) under similar tunneling parameters, and $3.5 \AA$ is an estimated height of a molecule lying down on $\mathrm{BN} / \mathrm{Cu}(111)$. Atomic force microscopy experiments were carried out in a Bruker Multimode 8 AFM in tapping mode under ambient conditions.

Molecular modeling. The MMFF parameter implementation in the program CHARMM c $36^{34}$ was employed for force field molecular dynamic (MD) simulations. For the in vacuo stacking studies, twelve simulations were performed in a cubic $125 \mathrm{~nm}^{3}$ cell with 10 molecules at temperatures between $370 \mathrm{~K}$ and $400 \mathrm{~K}$. The trajectories' $\pi$-stacking were analyzed by searching for $\mathrm{C}$ - $\mathrm{Br}$ carbons at a distance of less than $5 \AA$ from each other. This criterion much larger than the $\pi$-stacking distance was employed to account for displaced parallel stacking. For the studies on $\mathrm{BN}$, three layers of trans- $(1)_{2}$ (576 molecules) were initialized on a $\mathrm{BN}$ mimic $^{39}$ in a simulation cell of size $\mathrm{a}=127, \mathrm{~b}=132, \mathrm{c}=1000$. The Langevin thermostat was employed to perform constant temperature dynamics with a friction coefficient of $0.1 \mathrm{ps}^{-1}$ and a $1 \mathrm{fs}$ integration time step in vacuo, and $0.01 \mathrm{ps}^{-1}$ and $2 \mathrm{fs}$ on the substrate. Density-functional based tight-binding (DFTB) calculations were performed with the DFTB + package $^{35}$. DFTB is an approximate valence-only DFT method that employs localized atomic orbitals as basis functions. Carbon and oxygen were described by one $2 \mathrm{~s}$ and three $2 \mathrm{p}$ functions and hydrogen by a single 1s function as provided by the 3ob-3-1 parameter set ${ }^{65}$. Bromine was parameterized as described in ref. ${ }^{65}$. The Nosé-Hoover thermostat was employed for the DFTB-MD simulations with a coupling of $3000 \mathrm{~cm}^{-1}$ and a $0.5 \mathrm{fs}$ integration time step. Conjugated gradient geometry optimization search of optimized MD structures was performed using self-consistent charge third-order DFTB with either Lennard-Jones or Grimme dispersion approximation (see text). The self-consistency criterion was set to $10^{-5} \mathrm{e}$. The residual force on each atom was typically smaller than $10^{-3}$ a.u.

Scanning electron microscopy. The scanning electron microscopy (SEM) images were scanned by a HITACHI S4800 SEM under $1 \mathrm{kV}$ to $2 \mathrm{kV}$ high voltage, $6000 \times$ to $8000 \times$ magnification, respectively. We place the sample on a conductive tape on the holder, and in the SEM chamber with a base pressure of $10^{-7}$ mbar. $1 \mathrm{kV}$ was applied when the sample was not irradiated by the UV lamp, which avoids damage by the electron beam. Samples measured by SEM measured were not characterized further to avoid electron-irradiation related changes in the nanowires.

Raman spectroscopy. The $325 \mathrm{~nm}$ Raman spectra were recorded with a Horiba LabRam HR Evolution Raman spectrometer under $0.5 \mathrm{~mW} 325 \mathrm{~nm}$ laser, and the $532 \mathrm{~nm}$ Raman with a Renishaw inVia-Reflex Raman spectrometer under $2 \mathrm{~mW}$ $532 \mathrm{~nm}$ laser. Both Raman spectra were measured employing a $60 \mathrm{~s}$ integration time and $100 \times$ Olympus objective.

Photoluminescence microscopy and spectroscopy. The photoluminescence microscopy (PL) data was captured by an Olympus BX53 PL microscope, whose wavelength of irradiation lamp is $350 \mathrm{~nm}$. We employ a $100 \times$ Olympus objective to obtain the optical microscope image and PL microscope image of nanowires, and $5 \times$ Olympus objective to obtain the larger scale image of sample. The PL spectra were acquired in a homemade UHV chamber to monitor the process of selfassembly of 1, UV debromination and thermal conversion. The sample was irradiated by a $337 \mathrm{~nm}$ LTB MNL100 nitrogen laser operating at $\sim 80 \mathrm{~Hz}$. The signal was collected by a Princeton Instrument HRS-300 spectrometer and photomultiplier (PMT) detector. The HV of PMT was $1000 \mathrm{~V}$, and the integration time was $1000 \mathrm{~ms}$.

Matrix-assisted laser desorption ionization and time-of-flight mass spectrometry. A thin layer of (2E)-2-methyl-3-[4-(2-methyl-2-propanyl) phenyl]-2-propen-1-ylidene malononitrile (DCTB) was deposited on top of the samples. A $337 \mathrm{~nm}$ laser (LTB MNL100) was employed to ionize and desorb the molecules ${ }^{24}$. The matrix-assisted laser desorption ionization time-of-flight (MALDI-ToF) mass spectrometry (MS) experiments were carried out in a Bruker FLEX2 spectrometers under a pressure of $10^{-6}$ mbar. The MS of 1 was also measured by a Kore Tech electron ionization ToF-MS after deposition onto a BN/mica substrate. Species 1 was evaporated on $\mathrm{BN} / \mathrm{mica}$ substrate and the sample was set in front of the skimmer of the ToF-MS. The acquisition time was $30 \mathrm{~min}$, the ionization energy $10 \mathrm{eV}$ and the emission current $200 \mu \mathrm{A}$. During the measurement, the temperature of sample was $30^{\circ} \mathrm{C}$ at a pressure of $4 \times 10^{-9}$ mbar.

Electrical characterization. An Imina Micro Probe Station and Keithley 4200ASCS Parameter Analyzer were employed for electrical characterization of the samples. The samples were exposed to air during transfer from UHV and measured under argon unless stated otherwise, and usually driven for one minute at $10 \mathrm{mV}$ to stabilize the conductivity.

\section{Data availability}

All data supporting the findings of this work are available within this paper and its Supplementary Information. Raw data are available from the corresponding author upon request or will be made available at http://palmalab.org/publications.html.

Received: 3 June 2021; Accepted: 17 November 2021; Published online: 21 January 2022 


\section{References}

1. August, D. P. et al. Self-assembly of a layered two-dimensional molecularly woven fabric. Nature 588, 429-435 (2020).

2. Dou, J.-H. et al. Atomically precise single-crystal structures of electrically conducting 2D metal-organic frameworks. Nat. Mater. 20, 222-228 (2021)

3. Palma, C.-A., Samorì, P. \& Cecchini, M. Atomistic simulations of $2 \mathrm{D}$ bicomponent self-assembly: from molecular recognition to self-healing. J. Am. Chem. Soc. 132, 17880-17885 (2010).

4. Roussel, T. J., Barrena, E., Ocal, C. \& Faraudo, J. Predicting supramolecular self-assembly on reconstructed metal surfaces. Nanoscale 6, 7991-8001 (2014).

5. Spijker, P. et al. Understanding the interface of liquids with an organic crystal surface from atomistic simulations and AFM experiments. J. Phys. Chem. C. 118, 2058-2066 (2014)

6. Palma, C.-A. et al. Visualization and thermodynamic encoding of singlemolecule partition function projections. Nat. Commun. 6, 6210 (2015).

7. Whitelam, S. \& Jack, R. L. The statistical mechanics of dynamic pathways to self-assembly. Ann. Rev. Phys. Chem. 66, 143-163 (2015).

8. Packwood, D. M., Han, P. \& Hitosugi, T. Chemical and entropic control on the molecular self-assembly process. Nat. Commun. 8, 14463 (2017).

9. Klappenberger, F. et al. On-surface synthesis of carbon-based scaffolds and nanomaterials using terminal alkynes. Acc. Chem. Res. 48, 2140-2150 (2015).

10. Palma, C. A. in Encyclopedia of interfacial chemistry (ed. Klaus Wandelt) 361-369 (Elsevier, 2018).

11. Briseno, A. L., Mannsfeld, S. C. B., Jenekhe, S. A., Bao, Z. \& Younan, X. Introducing organic nanowire transistors. Mater. Today 11, 38-47 (2008)

12. Hartgerink, J. D., Beniash, E. \& Stupp, S. I. Self-assembly and mineralization of peptide-amphiphile nanofibers. Science 294, 1684-1688 (2001).

13. Aida, T. \& Meijer, E. W. Supramolecular polymers - we've come full circle. Isr. J. Chem. 60, 33-47 (2020).

14. Yao, Y. et al. Carbon welding by ultrafast Joule heating. Nano Lett. 16, 7282-7289 (2016).

15. Tang, C. et al. Long-range ordered thin films of block copolymers prepared by zone-casting and their thermal conversion into ordered nanostructured carbon. J. Am. Chem. Soc. 127, 6918-6919 (2005).

16. Xin, G. et al. Highly thermally conductive and mechanically strong graphene fibers. Science 349, 1083-1087 (2015).

17. Okawa, Y. \& Aono, M. Materials science - nanoscale control of chain polymerization. Nature 409, 683-684 (2001).

18. Palma, C.-A. \& Samorì, P. Blueprinting macromolecular electronics. Nat. Chem. 3, 431-436 (2011)

19. Lindner, R. et al. Substrate templating guides the photoinduced reaction of C-60 on calcite. Angew. Chem. Int. Ed. 53, 7952-7955 (2014).

20. Basagni, A. et al. Stereoselective photopolymerization of tetraphenylporphyrin derivatives on $\operatorname{Ag}(110)$ at the sub-monolayer level. Chem. Eur. J. 20, 14296-14304 (2014).

21. Gao, H. Y. et al. Photochemical glaser coupling at metal surfaces. J. Phys. Chem. C. 118, 6272-6277 (2014)

22. Grossmann, L. et al. On-surface photopolymerization of two-dimensional polymers ordered on the mesoscale. Nat. Chem. 13, 730-736 (2021).

23. Murray, D. J. et al. Large area synthesis of a nanoporous two-dimensional polymer at the air/water interface. J. Am. Chem. Soc. 137, 3450-3453 (2015).

24. Palma, C.-A. et al. Photoinduced C-C reactions on insulators toward photolithography of graphene nanoarchitectures. J. Am. Chem. Soc. 136, 4651-4658 (2014).

25. Liu, W. et al. A two-dimensional conjugated aromatic polymer via C-C coupling reaction. Nat. Chem. 9, 563-570 (2017).

26. Zhou, Y., Sreekala, S., Ajayan, P. M. \& Nayak, S. K. Resistance of copper nanowires and comparison with carbon nanotube bundles for interconnect applications using first principles calculations. J. Phys. Condens. Matter 20, 095209 (2008).

27. Borchardt, L. et al. Toward a molecular design of porous carbon materials. Mater. Today 20, 592-610 (2017).

28. Li, Z., Liu, Z., Sun, H. \& Gao, C. Superstructured assembly of nanocarbons: fullerenes, nanotubes, and graphene. Chem. Rev. 115, 7046-7117 (2015).

29. Yamamoto, T. \& Takimiya, K. Facile synthesis of highly pi-extended heteroarenes, dinaphtho[2,3-b: 2 ',3'-f] chalcogenopheno[3,2-b] chalcogenophenes, and their application to field-effect transistors. J. Am. Chem. Soc. 129, 2224 (2007).

30. Sokolov, A. N. et al. From computational discovery to experimental characterization of a high hole mobility organic crystal. Nat. Commun. 2, 437 (2011).

31. Cai, J. M. et al. Atomically precise bottom-up fabrication of graphene nanoribbons. Nature 466, 470-473 (2010).

32. Bieri, M. et al. Two-dimensional polymer formation on surfaces: insight into the roles of precursor mobility and reactivity. J. Am. Chem. Soc. 132, 16669-16676 (2010).
33. Björk, J., Stafström, S. \& Hanke, F. Zipping up: cooperativity drives the synthesis of graphene nanoribbons. J. Am. Chem. Soc. 133, 14884-14887 (2011).

34. Brooks, B. R. et al. CHARMM: the biomolecular simulation program. J. Comput. Chem. 30, 1545-1614 (2009).

35. Hourahine, B. et al. DFTB + , a software package for efficient approximate density functional theory based atomistic simulations. J. Chem. Phys. 152, 124101 (2020).

36. Grimme, S., Antony, J., Ehrlich, S. \& Krieg, H. A consistent and accurate ab initio parametrization of density functional dispersion correction (DFT-D) for the 94 elements H-Pu. J. Chem. Phys. 132, 154104 (2010).

37. Björk, J. et al. Adsorption of aromatic and anti-aromatic systems on graphene through pi-pi stacking. J. Phys. Chem. Lett. 1, 3407-3412 (2010).

38. Berland, K. et al. van der Waals forces in density functional theory: a review of the vdW-DF method. Rep. Prog. Phys. 78, 066501 (2015).

39. Palma, C.-A. et al. Two-level spatial modulation of vibronic conductance in conjugated oligophenylenes on boron nitride. Nano Lett. 15, 2242-2248 (2015).

40. Joshi, S. et al. Boron nitride on $\mathrm{Cu}(111)$ : an electronically corrugated monolayer. Nano Lett. 12, 5821-5828 (2012).

41. Moré, R. et al. Photodimerization of crystalline 9-anthracenecarboxylic acid: a nontopotactic autocatalytic transformation. J. Phys. Chem. C. 114, 4142-4148 (2010).

42. Shuai, Z. G., Geng, H., Xu, W., Liao, Y. \& Andre, J. M. From charge transport parameters to charge mobility in organic semiconductors through multiscale simulation. Chem. Soc. Rev. 43, 2662-2679 (2014).

43. Götzen, J., Kafer, D., Wöll, C. \& Witte, G. Growth and structure of pentacene films on graphite: weak adhesion as a key for epitaxial film growth. Phys. Rev. B 81, 085440 (2010)

44. Ambrosch-Draxl, C., Nabok, D., Puschnig, P. \& Meisenbichler, C. The role of polymorphism in organic thin films: oligoacenes investigated from first principles. N. J. Phys. 11, 125010 (2009).

45. Kratzer, M. \& Teichert, C. Thin film growth of aromatic rod-like molecules on graphene. Nanotechnology 27, 292001 (2016).

46. He, D. W. et al. Two-dimensional quasi-freestanding molecular crystals for high-performance organic field-effect transistors. Nat. Commun. 5, 5162 (2014).

47. Vogel, J.-O. et al. Phase-separation and mixing in thin films of co-deposited rod-like conjugated molecules. J. Mater. Chem. A 20, 4055-4066 (2010).

48. Salzillo, T., Venuti, E., Della Valle, R. G. \& Brillante, A. Solid-state photodimerization of 9-methyl-anthracene. J. Raman Spectrosc. 48, 271-277 (2017).

49. Ferrari, A. C. et al. Raman spectrum of graphene and graphene layers. Phys. Rev. Lett. 97, 187401 (2006).

50. Verzhbitskiy, I. A. et al. Raman fingerprints of atomically precise graphene nanoribbons. Nano Lett. 16, 3442-3447 (2016).

51. Ferrari, A. C. \& Robertson, J. Resonant Raman spectroscopy of disordered, amorphous, and diamondlike carbon. Phys. Rev. B. 64, 075414 (2001).

52. Wu, J. B., Lin, M. L., Cong, X., Liu, H. N. \& Tan, P.-H. Raman spectroscopy of graphene-based materials and its applications in related devices. Chem. Soc. Rev. 47, 1822-1873 (2018).

53. Dervishi, E., Ji, Z., Htoon, H., Sykora, M. \& Doorn, S. K. Raman spectroscopy of bottom-up synthesized graphene quantum dots: size and structure dependence. Nanoscale 11, 16571-16581 (2019).

54. Narita, A., Wang, X.-Y., Feng, X. \& Müllen, K. New advances in nanographene chemistry. Chem. Soc. Rev. 44, 6616-6643 (2015).

55. Che, G., Lakshmi, B. B., Martin, C. R., Fisher, E. R. \& Ruoff, R. S. Chemical vapor deposition based synthesis of carbon nanotubes and nanofibers using a template method. Chem. Mater. 10, 260-267 (1998).

56. Gu, H., Li, Y. \& Li, N. Electrical conductive and structural characterization of electrospun aligned carbon nanofibers membrane. Fibers Polym. 16 2601-2608 (2015)

57. Zhang, Q. et al. Transparent and freestanding single-walled carbon nanotube films synthesized directly and continuously via a blown aerosol technique. Adv. Mater. 32, 2004277 (2020).

58. Baringhaus, J. et al. Exceptional ballistic transport in epitaxial graphene nanoribbons. Nature 506, 349-354 (2014)

59. Aprojanz, J. et al. Ballistic tracks in graphene nanoribbons. Nat. Commun. 9, 4426 (2018)

60. Kockert, M. et al. Nanometrology: absolute seebeck coefficient of individual silver nanowires. Sci. Rep. 9, 20265 (2019).

61. Murali, R., Yang, Y., Brenner, K., Beck, T. \& Meindl, J. D. Breakdown current density of graphene nanoribbons. Appl. Phys. Lett. 94, 243114 (2009).

62. Kalinin, S. V., Borisevich, A. \& Jesse, S. Fire up the atom forge. Nature 539, 485-487 (2016)

63. Garcia, A. G. F. et al. Effective cleaning of hexagonal boron nitride for graphene devices. Nano Lett. 12, 4449-4454 (2012). 
64. Nečas, D. \& Klapetek, P. Gwyddion: an open-source software for SPM data analysis. Open Phys. 10, 181-188 (2012).

65. Kubillus, M., Kubař, T., Gaus, M., Rezáć, J. \& Elstner, M. Parameterization of the DFTB3 method for $\mathrm{Br}, \mathrm{Ca}, \mathrm{Cl}, \mathrm{F}, \mathrm{I}, \mathrm{K}$, and $\mathrm{Na}$ in organic and biological systems. J. Chem. Theory Comput. 11, 332-342 (2015).

\section{Acknowledgements}

This work was partly funded by the National Natural Science Foundation of China (no. 11974403, no. 21902012 and Sino-German Project no. 51761135130), the Chinese Academy of Sciences (nos. QYZDBSSW-SLH038, XDB33000000, XDB33030300), the Max Planck Society, the European Commission through the Graphene Flagship and the FET Open 2D Ink Project (no. 664878). F.G. and E.L.-K. gratefully acknowledge financial support by the Deutsche Forschungsgemeinschaft (no. 182087777 - SFB 951), and work conducted in the framework of the Joint Lab GEN_FAB. We gratefully acknowledge financial support from the Alexander von Humboldt Foundation (C.-A.P.). We are grateful to Guangyu Zhang, Jiawei Li for help with preliminary probe station measurements, and to Nedjla Tazdait, Hans Joachim Räder, Shixuan Du, Hong-Jun Gao for MS and AFM support. We thank Knud Seufert, Karl Eberle for UHV equipment support and Willi Auwärter for a critical reading of our manuscript. K.M. thanks the Max Planck Society for an Emeritus Group and the Gutenberg Research College for a Scholarship.

\section{Author contributions}

X.Z. measured the Raman, microscopy, SEM and PL spectroscopy. X.Z., Z.W., Y.W. and C.-A.P. measured the MS and scanning probe data. F.G. and E.L.-K. measured the electrical properties. Y.H. and A.N. prepared the precursor molecule. X.Z., Z.C., J.V.B, K.M. and C.-A.P. prepared the manuscript. J.L., J.C., L.X., A.N., K.M., J.V.B. contributed with experimental infrastructure and co-supervised the project C.-A.P. designed the research and supervised the project. All authors commented on the manuscript.

\section{Competing interests}

The authors declare no competing interests.

\section{Additional information}

Supplementary information The online version contains supplementary material available at https://doi.org/10.1038/s41467-021-27600-1.

Correspondence and requests for materials should be addressed to Carlos-Andres Palma.

Peer review information Nature Communications thanks Zhenyu Li and the other, anonymous, reviewer(s) for their contribution to the peer review of this work. Peer reviewer reports are available.

Reprints and permission information is available at http://www.nature.com/reprints

Publisher's note Springer Nature remains neutral with regard to jurisdictional claims in published maps and institutional affiliations.

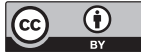

Open Access This article is licensed under a Creative Commons Attribution 4.0 International License, which permits use, sharing, adaptation, distribution and reproduction in any medium or format, as long as you give appropriate credit to the original author(s) and the source, provide a link to the Creative Commons license, and indicate if changes were made. The images or other third party material in this article are included in the article's Creative Commons license, unless indicated otherwise in a credit line to the material. If material is not included in the article's Creative Commons license and your intended use is not permitted by statutory regulation or exceeds the permitted use, you will need to obtain permission directly from the copyright holder. To view a copy of this license, visit http://creativecommons.org/ licenses/by/4.0/.

(C) The Author(s) 2022 